\title{
Interpretation of tail-pinch behaviors
}

\author{
LEE S. WESLER and ALLAN H. FREY \\ Randomline, Inc., Huntingdon Valley, Pennsylvania 19006
}

\begin{abstract}
Tail-pressure behavior (TPB) is increasingly being used as a dependent variable in neurochemical and behavioral experiments. We found that tail pressure, odor cues, schedule of testing, dependent variable used, and testing environment are significant factors to be controlled in such experiments and are often not controlled. Interpretations and conclusions from TPB experiments should thus be considered with caution.
\end{abstract}

Recently, numerous investigators have used as a dependent variable tail-pressure behaviors (TPB), defined as behaviors such as gnawing and licking elicited from a rat when a mild pinch is applied and maintained on its tail. TPB is increasingly being used as a dependent variable in experiments, and conclusions regarding behavior and neural function are being based on the results obtained.

Many methods have been used to induce TPB. An insulated surgical hemostat has been used to produce pressure on the rat's tail in several experiments (Antelman \& Szechtman, 1975; Antelman, Szechtman, Chin, \& Fisher, 1975; Nemeroff, Osbahr, Bissette, Jahnke, Lipton, \& Prange, 1978; Rowland, Marques, \& Fisher, 1980); a padded paper clip has been utilized as the tailpressure device (Koob, Fray, \& Iverson, 1976; Robbins, Phillips, \& Sahakain, 1977; Sahakian \& Robbins, 1977); "a length of electrical masking tape $(3.8 \mathrm{~cm}) 1 \mathrm{~cm}$ wide, wrapped firmly around the tail" has been used (Sahakian \& Robbins, 1977, p. 54); and "a specially designed cord noose . . . constricted until" certain behaviors were displayed has been used (Szechtman, Siegel, Rosenblatt, \& Komisaruk, 1977, pp. 807-808). Most of these reports omit information such as the amount of pressure per unit area exerted on the tail and whether the tail-pressure device was suspended so that the rat had freedom of movement. Testing was conducted in a variety of containers, such as shallow bowls (Antelman \& Szechtman, 1975; Antelman et al., 1975; Nemeroff et al., 1978; Rowland et al., 1980), and in open fields (Koob et al., 1976; Robbins et al., 1977; Sahakian \& Robbins, 1977). The behaviors used as dependent variables included eating of food pellets, gnawing, licking, "clip-directed behavior," and maternal behavior (Antelman \& Szechtman, 1975; Antelman et al., 1975; Koob et al., 1976; Robbins et al., 1977; Rowland et al., 1980; Sahakian \& Robbins, 1977; Szechtman et al., 1977).

Our experimentation with TPB led to our finding that certain controls and procedures are necessary to obtain reliable and interpretable results. In this report, we will briefly describe the TPB technique we developed out of the earlier work and our own experimentation, define controls and procedures that we found to be necessary, and discuss the results in the context of the findings and conclusions of earlier experiments.

\section{METHOD}

As a tail-pinch device, we adapted a plastic clothespin, modified by removing the metal parts and replacing them with rubber bands (see Figure 1). This provided a tail-pinch device that allowed us to regulate and quantify the pressure in grams per square millimeter over a $10-\mathrm{mm}^{2}$ area of tail. The pressure was applied a standard $4 \mathrm{~cm}$ from the tip of the tail, a point marked by an indelible pen. A box to contain the rat was developed that allowed the experimenter a clear view of the behavior, allowed feces to drop through so that they were not gnawed, and controlled the animal's position so that TPB could be reliably scored.

The box was $20.3 \times 20.3 \times 30.5 \mathrm{~cm}$ high, clear acrylic, with a floor consisting of $.63-\mathrm{cm}$-diameter acrylic rods, $1.5 \mathrm{~cm}$ on centers. Gnawing was on a wooden dowel rod mounted through a hole in a side wall near the front wall. This movable $1.2-\mathrm{cm}$ diameter rod projected into the box $9 \mathrm{~cm}$. It was held in place by a piece of foam rubber glued to the outside wall. The tailpressure device was suspended above the rat by rubber bands

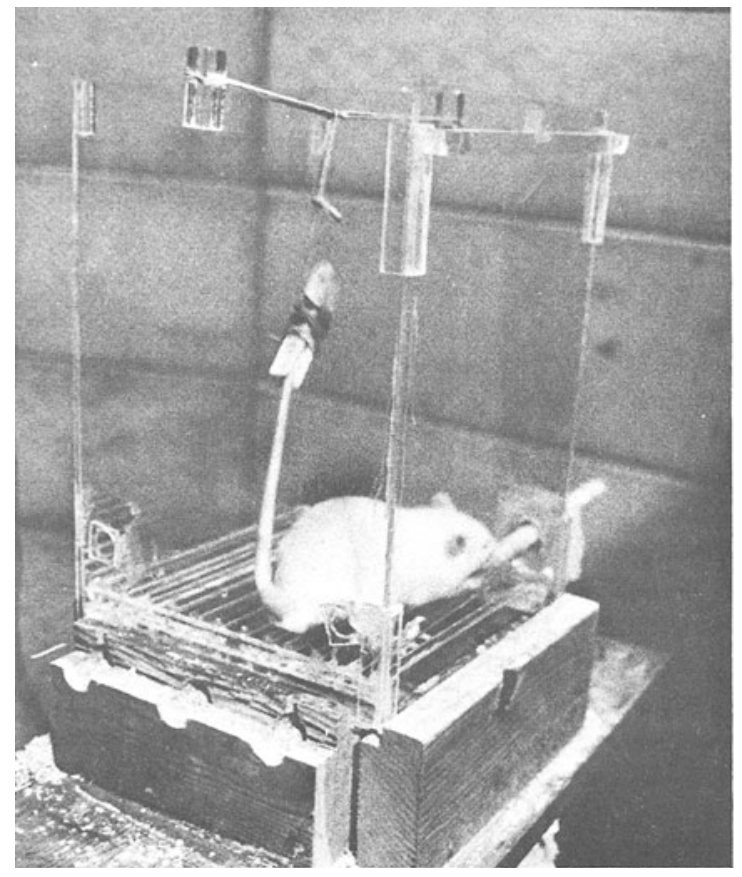

Figure 1. Animal in box with TPB device applied. 
Table 1

Effects of Pressure and Odor Cues on TPB

\begin{tabular}{cccc}
\hline & \multicolumn{4}{c}{ Median Time (in Seconds) } \\
\cline { 2 - 4 } Group & $\mathrm{G}$ & $\mathrm{L}$ & Total TPB \\
\hline A $\left(30 \mathrm{~g} / \mathrm{mm}^{2}\right.$, no odor cues) & 0 & 18 & 35 \\
B $\left(40 \mathrm{~g} / \mathrm{mm}^{2}\right.$, no odor cues) & $32^{*}$ & 20 & $58^{* *}$ \\
C $\left(30 \mathrm{~g} / \mathrm{mm}^{2}\right.$, with odor cues) & $16^{*}$ & $36^{*}$ & $73 \dagger$ \\
\hline
\end{tabular}

Note-Male Sprague-Dawley rats were tested with the technique and apparatus described in the text. The Mann-Whitney $U$ test was used to compare Groups $B$ and $C$ with Group $A$. The total $T P B$ median does not equal the sum of the gnawing (G) and licking $(L)$ medians because it was derived from the raw data. ${ }^{*} p<.05 . \quad{ }^{* *} p<.01 . \quad t p<.001$.

attached to the top of the box in a $\mathrm{T}$ configuration to allow the animal freedom of movement without access to the tailpressure device. An observer, who did not know the conditions of the experiment, recorded the duration of gnawing and the duration of licking. Total TPB (gnawing time plus licking time) could be calculated. We found these to be dependent variables that can be easily quantified and are characteristic TPB.

Male Sprague-Dawley rats were used. They were fed and watered ad lib. In one set of experiments, each rat was given three or four trials, one per day, depending on the particular experiment. A trial consisted of $1 \mathrm{~min}$ in which the rat was in the box without the tail pressure, followed by a 2 -min application of the tail pressure, during which the behaviors were recorded. In a second set of experiments, each rat was given five 2-min trials per day, separated by 18 -min intervals during an $83-\mathrm{min}$ run.

\section{RESULTS}

The amount of tail pressure significantly affected the type and duration of the behaviors displayed by the rat. There were a continuum of behaviors, with transitions in behaviors occurring as a function of tail pressure. Below about $20 \mathrm{~g} / \mathrm{mm}^{2}$ of pressure exerted over $10 \mathrm{~mm}^{2}$ of tail surface, no TPB was observed. In the zone of about $25 \mathrm{~g} / \mathrm{mm}^{2}$, rats showed primarily licking behavior. In the zone of about $40 \mathrm{~g} / \mathrm{mm}^{2}$, rats showed significantly more gnawing $(p<.05$, Mann-Whitney U test) and decreased licking, with a significant overall increase in the total TPB time ( $<.01$, Mann-Whitney U test) when compared to a $30-\mathrm{g} / \mathrm{mm}^{2}$ group (see Table 1). In the zone of about $80 \mathrm{~g} / \mathrm{mm}^{2}$, squealing sometimes occurred and aggressive behavior was seen when two animals were in the box at the same time.

Apparently, odor cues also significantly influence TPB. When odor cues from the urine and feces of previously tested animals were present, there was a relative increase in gnawing, decrease in licking, and increase in total TPB, as shown in Table 1.

Our experimentation showed that the schedule of testing was another important variable. We found that the duration of TPB displayed differed significantly over an 83-min period, during which five trials were given $(\mathrm{F}=6.77, \mathrm{p}<.001)$.

\section{DISCUSSION}

In the published reports, tail pressure is generally unspecified (e.g., several studies used surgical hemostats whose unknown pressure was probably quite high). Further, transient higher pinch pressure will occur on application and release of a hemostat. The effect of the drag of the relatively heavy hemostat is also unknown. Other studies have used a padded paper clip or a cord noose to apply pressure, the intensity of which is unknown. Thus, most of the published reports do not provide critical information needed to interpret the data, since there are qualitative and quantitative differences in TPB as a function of the nature and amount of the pressure.

Odor cues appear to enhance the tail-pressure stimulus. Yet, in the reports that use TPB, this has been an uncontrolled and, from the description of the apparatus (small surgical bowls to large open fields), a variable factor. Our experimentation indicates that the apparatus should be washed after use by each animal. In fact, our observation of TPB, apparently a mild stress behavior, suggests that washing the apparatus after each animal should be considered in all stress experiments.

The behaviors measured and the measurement criteria constitute another facet of TPB that is not consistent among experiments and is not well specified. Eating by nondeprived rats is a commonly reported dependent variable, but it was measured in different ways. Some investigators, for example, measured the weight of food consumed (Nemeroff et al., 1978). But we find that rats in TPB experiments tend to simply shred food and do not eat it. This is one of the factors that led us to use a wooden dowel rod. Another measurement technique was to count the number of jaw movements (Nemeroff et al., 1978). But the rat's jaw is not always in view, and there can be jaw movements even when food is not being chewed. Often, jaw movement is too rapid to count. The foregoing uncertainties are exacerbated by use of open fields, since the animal is often observed at a distance and has freedom to turn its back to the observer.

Tail pressure, odor cues, schedule of testing, dependent variable used, and testing environment are all significant factors to be contolled in the use of TPB techniques. Interpretations and conclusions in the published reports that used the TPB technique must be considered with caution.

Our experiments suggest that other variables, such as strain used and sex, should also be considered and are factors that require study.

\section{REFERENCES}

Antelman, S. M., \& Szechtman, H. Tail pinch induces eating in sated rats which appears to depend on nigrostriatal dopamine. Science, 1975, 189, 731-733.

Antelman, S. M., Szechtman, H., Chin,..P., \& Fisher, A. E. Tail pinch induced eating, gnawing and liching behavior in rats: Dependence on the nigrostriatal dopamine system. Brain Research, 1975, 99, 319-337.

Koob, G. F., Fray, P. J., \& Iversen, S. D. Tail pinch stimulation: Sufficient motivation for learning. Science, 1976, 194, 637-639.

Nemeroff, C. B., Osbahr, A. J., Bissette, G., Jahnke, G., Lipton, M. A., \& Prange, A. J. Cholecystokinin inhibits tail pinch induced eating in rats. Science, 1978, 200, 793-794.

Robbins, T. W., Phillips, A. G., \& Sahakian, B. J. Effects of chlordiazepoxide on tail pinch induced eating in rats. Pharmacology, Biochemistry and Behavior, 1977, 6, 297-302.

Rowland, N., Marques, D. M., \& Fisher, A. E. Comparison of the effects of brain dopamine-depleting lesions upon oral behaviors elicited by tail pinch and electrical brain stimulation. Physiology \& Behavior, 1980, 24, 273-281.

Sahakian, B. J., \& Robbins, T. W. Isolation-rearing enhances tail pinch induced oral behavior in rats. Physiology \& Behavior, 1977, 18, 53-58.

Szechtman, R., Siegel, H. I., Rosenblatt, J. S., \& KomisaruK, B. R. Tail pinch facilitates onset of maternal behavior in rats. Physiology \& Behavior, 1977, 19, 807-809.

(Received for publication July 17, 1982.) 